Clio. Femmes, Genre, Histoire

3| 1996

Métiers. Corporations. Syndicalisme

\title{
Les coordinations, filles des années 1968
}

\section{Didier LESCHI}

\section{Q OpenEdition}

\section{Journals}

Édition électronique

URL : http://journals.openedition.org/clio/467

DOI : 10.4000/clio.467

ISSN : 1777-5299

Éditeur

Belin

Édition imprimée

Date de publication : 1 avril 1996

ISSN : 1252-7017

Référence électronique

Didier LESCHI, «Les coordinations, filles des années 1968 », Clio. Histoire, femmes et sociétés [En ligne], 3| 1996, mis en ligne le 01 janvier 2005, consulté le 01 mai 2019. URL : http:// journals.openedition.org/clio/467; DOI : 10.4000/clio.467

Ce document a été généré automatiquement le 1 mai 2019.

Tous droits réservés 


\title{
Les coordinations, filles des années 1968
}

\author{
Didier LESCHI
}

1 Les infirmières, lors d'un mouvement qui a fait date dans l'histoire de la profession, ont animé pendant 18 mois - de mars 1988 à novembre 1989 - la plus importante à ce jour des coordinations du monde salarié. Dans un milieu aux faibles traditions de luttes et d'organisation syndicale, où la soumission aux contraintes professionnelles a longtemps été justifiée par un discours sur la nature particulière d'un métier qu'on embrasse par vocation, à l'image d'autres métiers dits féminins, ce mouvement était en tout point remarquable. Plus encore que les coordinations de cheminots et de métallurgistes de la SNECMA nées dans le prolongement du mouvement étudiant de l'hiver $1986^{1}$.

2 Cette forme coordination, redécouverte au milieu des années 1980, a une histoire. Sa résurgence dans l'espace propre au syndicalisme salarié n'est pas le produit d'une nouveauté. Elle est liée à la transmission d'une expérience de mobilisation, en décalage avec les normes syndicales, qui apparaît dans les années 1968 au cours des manifestations de la jeunesse scolarisée. C'est en effet dans ce secteur que sont nées les coordinations et qu'elles sont devenues un élément essentiel du répertoire d'action collective des lycéens et des étudiants. D'une décennie à l'autre, à la faveur du jeu de la mémoire, - «la contestation conserve son propre souvenir, et le résultat d'une lutte joue sur le caractère de la suivante $»^{2}$ - la forme coordination a été réutilisée par les étudiants à chaque mobilisation d'ampleur nationale, et par les cheminots en 1986.

3 Dans le cas des infirmières, la dimension féminine, à défaut d'être explicitement féministe, a permis d'analyser leur mouvement comme "sexué », et la forme prise par la grève des infirmières aurait reflété le lien existant entre «la façon dont les femmes comprennent et mettent en œuvre la démocratie dans les luttes et la forme coordination $»^{3}$. D'autre part, l'hypothèse a aussi été émise que les coordinations apparaissent dans certains secteurs de l'espace laissé vacant par l'incapacité des syndicats à représenter des couches sociales précises comme «la nouvelle figure salariale (féminine) que les infirmières préfigurent $»^{4}$.

4 Cet article voudrait donc saisir la généalogie et l'historicité de la forme coordination et mettre à l'épreuve ces deux hypothèses, en particulier à propos de la coordination 
infirmière, celle d'un mouvement sexué et celle d'un mouvement de nouvelles couches salariées surdiplômées.

Une innovation lycéenne

6 L'apparition du mode d'action «coordination » est directement lié à la succession de mobilisations qu'a connue l'enseignement secondaire après Mai 1968. Ces mouvements de contestation ont eu des aspects multiples tant politiques que culturels. En premier lieu, celui du refus de la "vie du lycée», c'est-à-dire d'une reprise, considérée comme routinière, des cours. Pendant plusieurs années, le fonctionnement quotidien de l'institution scolaire a été perturbé, ce qui déplut non seulement aux administrations, mais aussi à une partie du corps enseignant.

7 Face aux souhaits, exprimés par les lycéens ${ }^{5}$, de ne plus être infantilisés et de pouvoir aborder au sein de l'enceinte scolaire tous les sujets de la vie sociale - des luttes ouvrières à la sexualité -, les administrations sont déstabilisées. Les initiatives de reprise en main paraissent d'ailleurs d'autant plus brusques ${ }^{6}$ que les pouvoirs publics demeurent pendant longtemps incapables d'adopter une ligne de conduite claire, entre le souhait d'olivier Guichard, successeur d'Edgar Faure à l'Éducation nationale, de voir « inventer un ordre nouveau qui ne soit pas le lycée caserne $»^{7}$, et l'intervention de Georges Pompidou, président de la République, invitant dans une intervention télévisée, le 19 mars 1971, son premier ministre et le ministre de l'Éducation nationale à rétablir « sans retard et avec compréhension l'ordre dans les lycées ». Un tassement de la contestation lycéenne est notable après la mise en place de la réforme Haby à partir de 1976. Mais en 1980 encore, l'exclusion pour activité militante demeure une réalité8.

C'est dans ce contexte, entre 1968 et 1973, que les premières coordinations apparaissent. Alors même que les lycées sont nombreux et dispersés et qu'il n'existe pas une tradition de représentation collective, la succession de mobilisations dote le groupe "jeune" d'une dimension politique. Se constitue un peuple lycéen, selon l'expression de Gérard Vincent ${ }^{9}$ , qui conteste l'ordre établi et remet en cause les modes de vie traditionnels. La maladresse des pouvoirs publics renforce la solidarité des lycéens avec les militants. C'est à l'occasion de l'arrestation de Gilles Guiot, après une manifestation du Secours rouge ${ }^{10}$ le 9 février 1971, que la forme coordination prend véritablement corps. Au moment où se disperse la manifestation, un élève des classes préparatoires du lycée Chaptal, Gilles Guiot, quitte le lycée pour regagner son domicile. Après un contrôle d'identité, il est arrêté par deux policiers qui prétendent reconnaître en lui un de leurs agresseurs. Le lendemain, en audience des flagrants délits, ce lycéen est condamné à six mois de prison dont trois avec sursis. Non seulement Gilles Guiot n'a pas participé à la manifestation, mais il est «apolitique », c'est-à-dire, dans le langage journalistique de l'époque, non membre ou sympathisant d'une organisation d'extrême gauche. Un mouvement de solidarité s'enclenche, d'autant plus fort que la victime n'est pas sujette à caution.

L'initiative formelle de la mise en place de la première coordination lycéenne revient, à l'occasion de cette mobilisation, à des militants lycéens du lycée Chaptal. Catalyseurs de l'émotion des lycéens et utilisant le réseau fourni par leurs groupements politiques, ils convoquent une assemblée générale de délégués des comités parisiens de soutien à Gilles Guiot qui se transforme en coordination. Cette structure popularise le mouvement, édite des tracts et prend l'initiative d'appeler à une manifestation, le mercredi 18 février, manifestation interdite par la préfecture de Police ce qui amplifie son écho médiatique. La coordination, dont les actions sont attentivement suivies convoque alors un sit-in, le jour du procès, au Quartier latin, place Saint-Michel à deux pas du Palais de justice. Gilles 
Guiot est relaxé le soir même. L'extrême gauche lycéenne, très divisée depuis la dissolution des Comités d'action lycéens ${ }^{11}$ (CAL) à la rentrée 1969, a trouvé ainsi non seulement un mode commun d'action, mais surtout le moyen d'une représentation collective capable de coordonner la mobilisation de lycées dispersés ${ }^{12}$. La nouveauté de la coordination réside dans la mise en place d'un système de délégation qui a été capable de construire une image collective où chacun a pu se reconnaître: lycéens simplement solidaires ou sympathisants d'extrême gauche soucieux de faire reculer la politique répressive des pouvoirs publics. C'est une représentation collective plus large que celle des spectres partidaires, capable de mettre, dans l'action, un terme aux divisions. Les réseaux militants lycéens la poursuivent en 1973 contre l'application de la loi Debré (votée en 1971), qui prévoit de supprimer les sursis automatiques des étudiants. A cette occasion est mis en place pendant plusieurs semaines, un réseau national efficace. La coordination lycéenne contre la loi Debré se dote d'un Comité central de grève, de structures locales et régionales, d'une plate-forme. Enfin, elle manifeste sa volonté de ne voir personne négocier à sa place avec les pouvoirs publics. Cette première coordination nationale d'un mouvement de grève assoit définitivement le modèle " coordination » qui devient dès lors le passage obligé de toutes les tentatives de mobilisation dans les lycées et les universités. La recherche généalogique de ce nouveau mode spécifique d'organisation et de mobilisation permet à ce point d'en préciser les contours qui dans les lycées et les universités ne varieront plus.

Tentative de définition

12 Une coordination peut être définie comme une forme d'organisation temporaire, faisant son apparition au cours de périodes de mobilisation sociale, et se donnant pour fonction d'organiser et de représenter, si possible sur le plan national, des personnes appartenant à une même catégorie socioprofessionnelle en vue d'une action collective. Toutes les coordinations se sont jusqu'à maintenant présentées comme des structures assurant l'unité des acteurs mobilisés par-delà les tendances politiques ou syndicales.

13 Forme d'organisation temporaire, une coordination se différencie de ce fait d'une organisation syndicale ou politique. Si elles se pérennisent, les coordinations changent de nature. Ce passage d'un état à l'autre est parfois tenté, mais demeure une opération difficile. A titre d'exemple, la coordination infirmière ne peut maintenir son unité dès lors qu'elle aborde le débat de sa transformation en organisation permanente de type syndical à partir de juin $1989^{13}$. Dans la jeunesse scolarisée cependant, deux cas de coordinations ont réussi leur transformation en organisation permanente en gardant l'influence acquise: la coordination des collèges d'enseignement technique (CET) ${ }^{14}$ en 1973 transformée par Lutte ouvrière en Ceux du technique et la coordination lycéenne de 1978 qui se transforme en Coordination permanente lycéenne (CPL). Au lendemain du mouvement lycéen de 1973, la CGT préoccupée par l'impact des coordinations dans la jeunesse, en particulier dans l'enseignement technique - considéré comme le lieu de formation des ouvriers qualifiés - met sur pied une Coordination permanente des CET qui se présente comme une organisation syndicale des élèves du technique, tout en jouant sur le terme coordination. Cette organisation est dissoute par la CGT en 1980.

14 Les coordinations regroupent des acteurs d'une même catégorie socioprofessionnelle au sens étroit du terme: elles se différencient en cela d'autres structures temporaires de mobilisation transcatégorielles ou multisectorielles qui ont existé dans l'histoire comme les conseils ouvriers ou les soviets ${ }^{15}$. Les tentatives de mise en place de coordinations 
intercatégorielles dans les années 1968 se sont révélées être des échecs. Chez les cheminots, par exemple, ce sont les agents de conduite, l'élite de la profession, qui ont donné naissance à une coordination plus dynamique que celle dite intercatégorielle des cheminots qui rassemblait avec quelques agents de conduite, des guichetiers et des catégories considérées comme moins nobles du rail. L'émergence des coordinations n'est pas un phénomène spontané, mais manifeste une volonté de subversion du répertoire traditionnel des organisations syndicales, et de leur fonction, par des groupes militants qui ont intérêt à la modification des rapports de force entre organisations au moment où l'état de crise $^{16}$ dans un secteur donné n'est plus maitrisé par les organisations traditionnelles. Ce n'est donc pas la faible structuration syndicale ou politique d'un espace social qui détermine la possibilité que puisse surgir une coordination - si le secteur de la santé est faiblement structuré par les syndicats, il n'en va pas de même à la SNCF -, mais l'existence d'une concurrence entre groupes militants. Dès que les organisations s'avèrent capables de canaliser les crises dans l'espace social qu'elles contrôlent, une coordination ne peut venir imposer une modification des règles du jeu. Les échecs des différentes tentatives de coordinations d'instituteurs ou l'absence de coordination chez les enseignants du secondaire sont là pour l'attester. De même, si une coordination peut se mettre en place dans une phase de mobilisation, elle ne peut remettre en cause le mode de structuration dans un secteur donné qu'à la faveur d'une intensification de la crise, de l'affaiblissement général des organisations et de l'évolution du rapport des forces. Il est remarquable cependant que les secteurs où sont apparues des coordinations aient connu antérieurement à la crise des processus de mobilisation partielle qui ébranlent le système d'organisation de ces secteurs.

15 La grève de l'hiver 1986/87 a débuté chez les cheminots au lendemain de la présentation par la direction de la SNCF d'un projet de restructuration de la grille des salaires et de suppression de diverses primes, dont la prime de saisie informatique, attribuée aux sédentaires des guichets de réservation. Elle est la suite d'une série de conflits qui ont agité la SNCF depuis 1980 et dont les plus importants sont les mouvements de grève (en 1981, 1983, 1985) contre la conduite à agent unique. Les organisations syndicales ont été incapables de trouver une position qui n'apparaisse pas comme un refus de la modernisation et qui en même temps empêche la mise en place de la conduite par un seul agent et la réduction des effectifs qui accompagne cette mesure. Enfin, il faut y ajouter la crise d'autorité de la direction de la SNCF, dont la légitimité et la compétence sont remises en cause à la suite d'une série d'accidents ferroviaires, mais dont les responsabilités pénales incombent aux agents de conduite concernés.

De même, le secteur hospitalier connaît, avant 1988, pour des raisons tant financières que statutaires, des mouvements de crise réguliers, dont les infirmières n'ont pas le monopole. Des médecins aux personnels non médicaux, chaque catégorie s'est mobilisée au cours des dernières années. Sans parler de la grève contre le ministre communiste J. Ralite, organisée en 1983 par les patrons pour la défense du secteur privé hospitalier, les crises hospitalières prennent la forme de mobilisations partielles, de protestations locales, sur les conditions de travail en particulier. Ces mobilisations ont été, un temps, plus ou moins contrôlées par les organisations syndicales dans le cadre de journées d'action régulières. C'est au lendemain d'une de ces journées d'action syndicales, en mars 1987, que débute le processus de construction de la coordination à l'initiative d'un groupe de militants minoritaires au sein de la CFDT. Elle est le produit des habitudes de travail entre des syndiqué-e-s de la CFDT et des non syndiqué-e-s, en particulier dans le secteur 
psychiatrique. En convoquant les premières réunions, le $\mathrm{CRC} / \mathrm{CFDT}^{17}$ a mobilisé autour de lui cette frange militante de non syndiqué-e-s qui a un rôle symbolique dépassant ses capacités militantes :

il faut déjà dire que, dès le mois de mars, quand il y a eu la manifestation du 25 organisée par l'UNASIIF (qui est l'association "corpo." des infirmières), ceux qui ont lancé, dans cette manif, un appel à une assemblée générale l'après-midi même étaient en général des syndiqués, et même des militants syndicaux. Cette assemblée a eu lieu à la Bourse du Travail. Après la discussion, un petit groupe a pris l'initiative de se réunir dès la semaine suivante et préparer la poursuite de l'action. Dans ce petit groupe [il s'agit du bureau provisoire, c'est nous qui soulignons] on était cinq... dont trois syndicalistes. ${ }^{18}$

17 La mise en place des coordinations pose le problème de la limite des organisations traditionnelles à être les uniques représentantes des groupes mobilisés. La réussite de la grève lancée par la coordination infirmière montre que les syndicats ne parviennent plus à canaliser la mobilisation dans un cadre défini et contrôlé par eux. Une vive lutte d'influence se déroule entre les tenants d'un mode revendicatif qui refuse de remettre en cause la structure globale, la hiérarchie de responsabilité et les rapports sociaux, sans que l'on puisse dire que ceux qui représentent une conception traditionnelle de l'action soient extérieurs au groupe social mobilisé. Il s'est trouvé des infirmières de la CFDT pour exclure les militantes et militants qui animaient la coordination des infirmières, comme des agents de conduite CGT pour condamner la forme du mouvement de 1986. Enfin, dès leur origine, les coordinations ont favorisé un mode revendicatif plus soucieux du qualitatif - le rapport au travail, à l'institution ou aux modes de vie et les rapports entre les sexes - que du quantitatif. Par son succès, la coordination des infirmières a sans doute permis d'asseoir cette spécificité des coordinations dans le monde salarié qui est de faire émerger des « revendications diffuses pour lesquelles le syndicat, même quand il est présent, n'est pas un canal possible (le malaise enseignant, l'intrication vie privée / vie professionnelle chez les conducteurs de train, certaines formes atypiques de revendications...) $»^{19}$. C'est dans la jeunesse cependant que cet aspect des coordinations a été le plus développé à la faveur des mobilisations des années 1970. Si le mouvement lycéen de 1973 a eu pour prétexte la loi Debré, son contenu revendicatif dépasse largement le problème des sursis qui du reste concernait moins les lycéens que les étudiants du reste peu mobilisés. Le mouvement de 1973 fait rejaillir des questions sur l'organisation scolaire, le contenu des cours et le mode de vie. Les thèmes féministes pénètrent au sein des lycées et le mouvement favorise la constitution des premières commissions ou groupes-femmes sous l'impulsion de lycéennes sensibles à ces thèmes. Elles développent une argumentation spécifique en faveur de la participation à la mobilisation contre la loi Debré: celui de la critique des travers phallocratiques de l'institution militaire. Là encore s'exprime à travers la coordination le refus de l'ordre moral très présent encore dans la société. Le rapport étroit entre sexualité, contraception et féminisme est à l'origine d'initiatives auxquelles les pouvoirs publics répondent par des sanctions. Au cours de l'année scolaire 1972/1973, une enseignante, professeur de philosophie dans un lycée de Belfort, est inculpée pour "outrage aux bonnes mœurs » pour avoir lu à ses élèves un tract du docteur Carpentier « apprenons à faire l'amour car c'est le chemin du bonheur ». Des groupes femmes se créent dans des lycées en particulier dans certaines grandes villes (Paris, Marseille, Lyon et Caen).

Chez les élèves du technique à partir de 1973, les comités et les coordinations demandent l'égalité d'accès entre garçons et filles à toutes les formations professionnelles, alors que 
les filles sont de fait interdites d'accès à certains secteurs particulièrement discriminants. Cette revendication se retrouve encore dans les coordinations de 1978 contre l'alternance; c'est un des motifs de distanciation entre la CGT et son organisation de collégiens - la Coordination permanente des CET/LEP - à la suite des difficultés rencontrées avec un groupe d'élèves imprimeurs qui admettent mal que les filles ne puissent travailler la nuit dans les métiers qualifiés du livre alors qu'elles le peuvent dans les métiers non qualifiés, comme aux Nouvelles Messageries de la Presse Parisienne. En 1976, la plate-forme de lutte de la coordination lycéenne contre la réforme Haby comporte un chapitre sur l'égalité d'accès à toutes les filières entre garçons et filles, visant explicitement les filières scientifiques. Il en est de même à l'Université. On retrouve cette préoccupation en 1986 dans les milieux militants. Sciemment, le bureau de la coordination fait apparaître deux porte-parole (une étudiante et un étudiant) afin de mieux représenter le milieu étudiant, à majorité féminin. Cette mesure, non remarquée à l'époque par la presse, soulignait le décalage entre le monde étudiant et ses représentants institutionnels. La MNEF, et les syndicats étudiants sont présidés ou dirigés par des hommes ${ }^{20}$. Grâce à l'impact de la coordination de 1986 et au rôle joué par des militantes, des étudiantes ont pu accéder, dans des partis politiques de gauche, à des responsabilités qui leur auraient été très certainement inaccessibles sans ce mouvement étudiant. C'est une des conséquences de ce mouvement d'avoir pour la première fois été représenté par des figures autres que masculines et d'avoir ainsi levé un blocage. Le mouvement lycéen qui s'est déroulé en 1989 a vu de la même manière apparaitre des figures féminines comme porte-parole de la mobilisation. Cela n'a pas été le cas en 1973 ou 1976. Peut-être serait-il intéressant de relier cette émergence des filles dans les mobilisations lycéennes à leur réussite scolaire supérieure à celle des garçons.

Dans les coordinations infirmières, on retrouve des personnes ayant connu les mobilisations de la jeunesse lycéenne et étudiante des années 1970. Ainsi lors d'un entretien accordé à la revue syndicale Collectif, Irène Leguay, du bureau de la coordination infirmière, indique : « Auparavant, il y a eu un conflit dont je me souviens en 1978, j'étais élève à l'école d'infirmières, on avait fait grève contre l'exploitation des élèves... ». Au sein du bureau de la coordination des infirmières à la fin de la grève nous trouvons plus de $25 \%$ de membres qui ont connu les coordinations d'élèves infirmiers, dont l'un des porte-parole, Pascal Dias ${ }^{21}$. Certains ont choisi, parfois même après avoir obtenu un diplôme de troisième cycle, de devenir infirmiers ou infirmières psychiatriques ${ }^{22}$. C'est donc dans les parcours individuels que se lit la spécificité d'une génération de femmes et d'hommes, de militantes et de militants, issus d'un double héritage des années 1968 : un emploi en décalage avec une formation initiale élevée.

Un double héritage : les années 68 et la crise économique

21 L'étude des coordinations de salariés montre que leur genèse est directement liée à l'action de groupes militants dont la caractéristique essentielle est d'être, à cause de leur expérience antérieure, en décalage avec les normes qui dominent le monde syndical : des directions chargées de définir les stratégies et des modes corrects d'action ainsi que des registres de revendications. En ce sens, les coordinations sont un fait de génération, non pas tant en terme de classe d'âge, même s'il n'est pas anodin que la moyenne d'âge autour de 33/34 ans au sein de la coordination infirmière ${ }^{23}, 32$ ans au sein d'une des coordinations cheminotes ${ }^{24}$ - de celles et ceux qui s'investissent dans les coordinations dans le monde salarié, indique qu'ils ont appartenu aux générations lycéennes des années 1970; mais au sens où il s'agit bien d'un groupe qui, quand il se mobilise, «éprouve 
comme naturelles ou raisonnables des pratiques ou des aspirations que les autres ressentent comme impensables ou scandaleuses ou inversement $\aleph^{25}$. Après avoir intégré les coordinations comme un instrument du répertoire d'action collective au cours des manifestations de la jeunesse scolarisée, les militants venus de ces mobilisations de la jeunesse ont continué à bousculer les modes d'action traditionnels des syndicats ${ }^{26}$. Face aux défenseurs de l'ordre, ils ont «l'habitus impliquant la connaissance et la reconnaissance des lois immanentes du jeu, des enjeux, etc. $»^{27}$, et la particularité d'avoir une compétence et la connaissance pratique qui permettent ainsi de produire le discours nécessaire pour organiser des mobilisations. De plus ils disposent en général d'un capital culturel supérieur à la moyenne de leur milieu. Ainsi une étude de l'INRP indiquait que « les militants d'extrême gauche, et en particulier ceux de la Ligue communiste ont des particularités qui renforcent leur aura: leurs connaissances culturelles, politiques et économiques sont beaucoup plus fortes que celles de leurs camarades $»^{28}$. Les mêmes différences de capital culturel se retrouvent entre salariés militants et non militants ${ }^{29}$. Les coordinations fonctionnent grâce à cette rencontre entre des acteurs qui expriment leurs mécontentement et des militants. Mais, elles renvoient aussi plus généralement aux spécificités de leurs secteurs d'activité.

Un point commun semble bien relier les différentes coordinations entre elles: le capital scolaire des acteurs mobilisés dans le cadre de ces coordinations, si l'on convient que « la propension à déléguer à d'autres, reconnus pour leur compétence technique, la responsabilité des affaires politiques varie en raison inverse du capital scolaire possédé » ${ }^{30}$. Toutes les catégories salariées qui ont connu des coordinations ont un capital scolaire ou de formation élevé. C'est vrai à la SNCF, tant pour les agents de conduite que pour les guichetiers sédentaires. C'est vrai aussi chez les infirmières. Ces catégories ont vu leurs compétences et qualifications augmenter et leurs fonctions évoluer en même temps que l'évolution des techniques. Que ce soit à la SNCF, où cette transformation est liée à celle du matériel, ou chez les infirmières (la spécialisation ne touche pas seulement les médecins, mais l'ensemble des personnels soignants). Ainsi les infirmières sont passées d'une qualification étroite, limitée aux soins mineurs (toilettes, pansements, simples injections) à l'adaptation aux évolutions techniques qui nécessitent une augmentation de la qualification. Les secteurs de la fonction publique, dont le niveau de recrutement est scolairement élevé, ont été les premiers touchés ; le recrutement par concours draine des surtitrés qui trouvent des débouchés dans des emplois autrefois destinés à des individus au capital scolaire inférieur. Au concours de recrutement des écoles d'infirmières se présentent des candidates qui ont souvent fait une ou deux années d'études supérieures, parfois plus. Les guichetiers SNCF ou les postiers sont parfois titulaires d'une licence. La présence de ces catégories permet d'expliquer le détachement vis-à-vis du mécanisme de la délégation syndicale dans les périodes de mobilisation. Des groupes d'acteurs se comportent comme si leur implication favorisait leur politisation et les amenait à vouloir prendre directement en charge une mobilisation qui les concerne et dont ils pensent être aptes à maîtriser la dynamique. Cette « assurance culturelle » résulte en particulier d'un passage prolongé dans le système d'enseignement. Le système éducatif est aussi à l'origine d'un comportement « citoyen » avec mise en place d'un instrument à vocation représentative, où la délégation se fait de manière contrôlée, aux dépens d'une délégation au responsable syndical qui apparaît d'autant plus lointain que son élection a en général suscité peu d'intérêt. Le vocabulaire mis en avant, en particulier par les étudiants, mais que l'on retrouve aussi chez les infirmières, reflète cette aspiration au contrôle: les notions de «mandat impératif », de «bureau » au départ presque toujours " provisoire » 
sont là pour le souligner et laissent en permanence la possibilité de remettre en cause ce qui est déjà installé.

Dans le secteur scolaire, les lycéens de 1973, comme de 1971, sont emmenés par des élèves de Terminales et de classes préparatoires. Dans le technique, les élèves en Brevet de Technicien Supérieur sont les premiers à se mobiliser, suivis des lycéens du technique et, en 1977 et 1978, par les élèves de BEP. De plus, la naissance des coordinations dans les lycées est liée à une phase importante de mobilisation politique qui augmente le nombre de ceux qui, grâce à l'action collective, acquièrent une assurance quelque soit leur âge, comme l'atteste le fait qu'en 1971 et surtout 1973 des élèves des classes de quatrième et de troisième (et même moins) aient pu participer à des coordinations.

Cependant, cette assurance a des degrés variables et les acteurs n'ont pas une politisation homogène. La place accordée parfois aux permanents syndicaux lors des périodes de crise en est la conséquence. L'intégration de représentants syndicaux est une réponse apportée à ces décalages de politisation, ce qui est particulièrement net dans le cas des agents de conduite de la SNCF. La coordination codifie la présence des représentants des organisations syndicales au sein de ses instances. Elle permet de rassurer ceux qui gardent leur confiance aux syndicats et aux mécanismes traditionnels de défense de leurs intérêts, sans mécontenter les autres.

Le haut niveau scolaire n'est cependant pas automatique pour les acteurs engagés dans la pratique des coordinations. Tout fonctionne comme si cela concernait au premier chef des groupes sociaux issus de ces générations, dont parle Pierre Bourdieu, qui sont victimes de cette "désillusion collective qui résulte du décalage structural entre les aspirations et les chances, entre l'identité sociale que le système d'enseignement semble promettre ou celle qu'il propose à titre provisoire et l'identité sociale qu'elle offre réellement, au sortir de l'école ». L'élément moteur des mobilisations dans les CET/LEP, jusqu'aux réformes permettant aux élèves de ces filières d'espérer un retour vers le secondaire et la perspective d'une entrée vers le supérieur, est formé d'élèves de BEP recrutés à la sortie de la seconde et en situation d'échec scolaire. La situation des élèves de BEP est d'autant plus frustrante et dévalorisée que les conventions collectives ne reconnaissent que les CAP. Dans les universités «ce sont les sections où les promotions professionnelles sont les plus réduites (où l'écart entre le rendement attendu du titre scolaire et les `situations' effectives offertes est le plus spectaculaire), dans les universités où les effectifs de ces sections sont les plus nombreux, à l'instar du département de sociologie de certaines universités parisiennes, qui fournissent les contingents d'avantgarde de la révolte étudiante ${ }^{31} »$. En 1986, la situation universitaire des porte-parole du mouvement, illustre une situation qui ne s'est pas modifiée. Au sein même des délégations présentes dans les coordinations, les étudiants qui représentent les universités sont en général des étudiants en lettres ${ }^{32}$, dont ce n'est pas la première expérience de mobilisation. Ce sont donc les catégories les plus en situation de frustration relative, ou qui prennent conscience de leur situation réelle au regard de leurs aspirations, qui sont à la pointe des mobilisations et constituent l'armature des coordinations. A la SNCF, ce sont deux des catégories les plus diplômées que l'ont retrouve en priorité au sein des coordinations. Les agents de conduite et les guichetiers agents commerciaux qui ont le bac et plus. A cela s'ajoute, dans le déroulement même de la carrière, le décalage entre le type de tâches et de responsabilités confiées réellement, et sa non-prise en compte par un statut défini au sein du processus de travail et conférant un pouvoir réel de décision. Ainsi, la SNCF se caractérise par un type d'organisation du 
travail autoritaire et bureaucratique. Dans la santé, les infirmières sont au point de jonction entre les malades et le corps médical, mais leur statut reste fondé sur la dépendance et la soumission, sans pouvoir décisionnel. A cela s'ajoute le sentiment d'une carrière bloquée, sans perspective de promotion sociale. Pour les roulants, à la SNCF, la diminution des effectifs liée à l'amélioration des techniques (la conduite par un agent seul) contribue à diminuer la possibilité de promotion interne par l'affectation sur des trains à longue distance et en particulier les TGV. Le déroulement des carrières des professions de santé est tout aussi bloqué ; leur évolution dépend de la reconnaissance d'un statut de l'infirmière qui corresponde aux tâches effectives ${ }^{33}$, reconnaissance qui ne peut se faire sans une remise en cause de la hiérarchie médicale.

Les groupes qui se mobilisent dans le cadre des coordinations sont donc ceux qui, par leur niveau scolaire, la compétence et la situation réelle, ne sont pas les plus démunis, mais s'estiment dévalués. Dans ce cadre, le refus de l'inter-catégories des coordinations s'explique aussi par le refus de lier son sort à celui d'autres dont la situation est moins enviable. Pour des catégories qui sont employées à un niveau de compétence inférieur à leur niveau scolaire, et qui ont tendance à être tirées vers le bas, lier leur sort aux catégories inférieures c'est aller dans le sens d'une non-reconnaissance de leur spécificité dans le processus de soins. Les infirmières ont avant tout sollicité la solidarité de personnalités ou de catégories supérieures à leur statut dont le poids symbolique soulignait leur importance, et ont eu vis-à-vis des catégories non soignantes une attitude de distanciation. Sans rejeter les revendications des autres catégories (aides-soignantes, brancardiers), elles se sont adressées aux médecins et grands patrons seuls à même de les reconnaître dans leur valeur médicale et sociale au sein de la structure hospitalière. De même les agents de conduite de la SNCF appellent l'ensemble des personnels à faire comme eux, mais en aucun cas à se joindre à eux. Ce rapport à l'intercatégoriel est un des éléments qui différencie les coordinations du syndicalisme confédéré justement censé élaborer les revendications en fonction d'une vision d'ensemble. Il est vécu comme stratégie de dilution des revendications, qui ne permet plus aux acteurs de contrôler leur mouvement. et comme stratégie de reconquête de la délégation de pouvoir par les professionnels syndicaux.

Le débat sur l'intercatégoriel n'est pas spécifique aux salariés. En 1973, le mouvement lycéen contre la loi Debré débute chez les élèves du technique. Ils sont à l'initiative de la première coordination à Paris, mais leurs problèmes spécifiques passent au second plan. Le sentiment d'être dépossédés de leur lutte aboutit à la mise en place de deux coordinations, les collégiens du technique en constituant une spécifique ${ }^{34}$. En 1986, la coordination étudiante, en monopolisant la représentation de la jeunesse «toutes catégories ", relègue au second plan la mobilisation des lycéens, numériquement plus forte que celle de leurs aînés ${ }^{35}$. Ainsi, se dessine une des caractéristiques de ces coordinations : l'unicité catégorielle où se différencient étudiants, lycéens et élèves du technique.

Bien que catégorielles, les coordinations sont une figure de la radicalité. Cependant cette radicalité, capacité à remettre en question le cours ordinaire des modes de revendication et de mobilisation, n'est pas nécessairement fonction du nombre de personnes impliquées. Elle dépend bien d'avantage de la mise en avant d'objectifs et de méthodes qui rompent le consensus revendicatif dans l'espace public. Il semble cependant que l'extension d'un mode d'action s'accompagne d'une forme de banalisation et d'affadissement, y compris pour les coordinations. Dans la jeunesse, les coordinations les 
plus marquantes sont celles qui ont posé des problèmes impertinents : en 1971, le droit à la citoyenneté et à la participation des jeunes de moins de 21 ans à la vie publique, récurrent depuis 1968; en 1973 le refus du retour à un ordre moral qui passe par un encadrement plus accentué des jeunes en cours d'études. Les coordinations de 1986 et celles qui ont suivi n'ont pas connu la même radicalité revendicative malgré la forte mobilisation qui les sous tendaient. L'apparition du thème politico-médiatique de "génération morale » en est un des effets. Un autre effet est l'emploi d'une forme singulière de normalisation des mouvements de jeunes: le dialogue avec des représentants choisis par les pouvoirs publics de ces mouvements précédant de peu leur intégration dans le jeu institutionnel. Jamais, à partir du milieu des années 1980, autant de jeunes issus des mobilisations n'auront été reçus par les autorités, y compris les plus hautes, installés dans des commissions ou dans des statuts de conseiller du prince, pour un résultat aussi peu important en terme de modification des comportements et des situations. Ce type de rapport n'a été rendu possible qu'en contrepartie d'un travail d'édulcoration des fonds revendicatifs réalisé par ceux qui contrôlaient ou qui assuraient la représentation de ces mouvements. D'où le malaise qui accompagne, par exemple, la fin des grèves de 1986. L'inflation médiatique est vécue comme un moyen de promotion de certains dirigeants et non comme le biais nécessaire pour répercuter le mal de vivre que l'on a voulu exprimer par la grève. Elle a accentué la perte de crédibilité des organisations syndicales étudiantes, en particulier de gauche ${ }^{36}$. La coordination infirmière n'a pas connu les mêmes problèmes de délitement et occupe une place originale. Elle a manifesté une capacité à rassembler et à marquer en imposant une figure salariale combative à dominante féminine qui n'a pas été affadie par l'intronisation de ses représentantes dans un théâtre institutionnel. Il est vrai que le refus du dialogue manifesté par les autorités ne favorisait pas une telle intronisation.

La coordination du mouvement des infirmières a une filiation avec les coordinations lycéennes des années 1968. Le bilan de la coordination infirmière et des autres coordinations indique néanmoins qu'elles ne sont pas, par nature, un instrument de subversion des appareils ou des modes revendicatifs traditionnels. Elles ne sont pas non plus l'apanage de nouveaux secteurs que les organisations syndicales seraient incapables de représenter, point de vue réducteur car les coordinations naissent dans des secteurs qui ne sont pas tous, à proprement parler, nouveaux; elles sont plutôt le produit de la défiance qui existe vis-à-vis de la forme traditionnelle de délégation de pouvoir et une recherche d'autres rapports de délégation. On peut penser qu'elles peuvent être la forme privilégiée d'une « nouvelle figure du salariat féminin » si sa définition accepte d'indiquer qu'il s'agit d'un salariat surqualifié par rapport à sa situation de travail et dans une situation de non-reconnaissance sociale de sa qualification. L'expérience montre que la forme coordination est plus adaptée à une action collective où les préoccupations qualitatives sont essentielles et surtout, où l'aspiration au contrôle de sa lutte participe du besoin de reconnaissance. L'expérience des coordinations chez les salariés demeure cependant trop limitée - alors qu'elle s'étale sur près de deux décennies dans les lycées et les universités - pour permettre une conclusion définitive sur l'apparition, avec elles, d'une nouvelle figure salariale sexuée. De plus, cette caractéristique spécifique du travail à majorité féminine - en particulier chez les infirmières - est, compte tenu de la situation économique, en passe de s'étendre à l'ensemble des salariés, hommes et femmes. Il n'est pas certain que le caractère très féminisé du salariat infirmier explique la forme coordination. En revanche, il est indéniable que cette forme favorise l'engagement et la 
prise de responsabilités des acteurs sociaux, et qu'elle a permis de souligner, dans le cas des infirmières, la capacité des femmes à intervenir dans l'espace public.

\section{BIBLIOGRAPHIE}

\section{ANWEILER Oskar}

1972 Les Soviets en Russie, Paris, Gallimard.

BOURDIEU Pierre,

1972 Esquisse d'une théorie de la pratique, Genève, Droz,.

1979 La Distinction, Paris, Minuit.

1980 Questions de sociologie, Paris, Minuit.

Collectif

1989 « Mouvement syndical et dynamique sociale », $n^{\circ} 7$, janvier.

Comités d'action lycéens

1969 Les Lycéens gardent la parole, Paris, Seuil.

Critique communiste

1989 « Le mouvement infirmière, la nouvelle vague ", $\mathrm{n}^{\circ} 78$.

DIETRICH François

1988 « Les racines d'un mouvement », Les Cahiers du féminisme.

DOBRY Michel

1986 Sociologie des crises politiques, Paris, Presses de la FNSP.

GAXIE Daniel

1978 Le Cens caché. Inégalités culturelles et ségrégation politique, Paris, Seuil .

GREBIL Rémy

1988 La Coordination nationale, ses causes et ses conséquences durant la grève des cheminots, mémoire sous la direction de Christian de Montlibert, université de Strasbourg.

INRP

1976 Les Lycéens, les mass-media et le monde contemporain, $n^{\circ} 83$.

KERGOAT Danièle (dir.)

1990 Les Infirmières et leur coordination, Gedisst/CNRS.

LACROIX Bernard

1987 L'Utopie communautaire, Paris, PUF.

LCR 
1988 La LCR et le mouvement syndical, politique et revendicatif, La Brèche.

\section{LESCHI Didier}

1986 1968-73, 5 ans de contestation dans les lycées, mémoire de maîtrise d'histoire contemporaine, Paris X.

1989 Un aspect particulier des mobilisations : les coordinations, étude comparée du phénomène des coordinations, mémoire de DEA de politique comparée, Paris X Nanterre,

LESCHI Didier et BENNHAMIAS Jean-Luc

1987 « La déferlante lycéenne », in Notre printemps en hiver, Assouline David et Zappi Sylvia (dir.), Paris, La Découverte.

RIBEILL Georges

1987 « Quand la SNCF déraille... Enquête sur les circonstances et les responsabilités d'un accident exceptionnel », Travail, octobre.

TILLY Charles

1984 « Les origines du répertoire de l'action collective contemporaine en France et en Grande Bretagne ", Vingtième siècle, $\mathrm{n}^{\circ} 4$, octobre.

VINCENT Gérard

1974 Le Peuple lycéen, Paris, Seuil.

\section{NOTES}

1. Leschi Didier 1989.

2. Tilly Charles 1984

3. Kergoat Danièle $1990: 121$.

4. Ibid : 11 .

5. CAL 1969. Rappelons que, jusqu'en 1974, la majorité légale est à 21 ans.

6. Le Monde du 26 novembre 1971 évoque « une épuration effectuée par les administrations selon une procédure parfois très contestable qui atteint un nombre de lycéens dont nul n'est en mesure de donner le chiffre précis ». Par exemple, au lycée Turgot à Paris, 206 élèves sont exclus en fin d'année scolaire 1970-1971, cf Didier Leschi 1986.

7. Le Monde, 11 juillet 1969.

8. Le Monde, 1er octobre 1981.

9. Vincent Gérard 1974 .

10. Le Secours rouge, dont le nom faisait référence à l'organisation de défense des militants de l'Internationale communiste dans les années 1930, a été créé à l'automne 1970 à la suite de l'emprisonnement d'Alain Geismar et de nombreux militants de la Gauche prolétarienne, groupe maoïste dissous le 27 mai 1970 par le gouvernement à la suite de violentes manifestations au Quartier latin. Le Secours rouge regroupait à son origine le PSU et l'ensemble des groupes d'extrême gauche (à l'exception des trotskystes de l'AJS), et était parrainé, notamment, par Jean-Paul Sartre et Charles Tillon, ancien dirigeant du PCF.

11. Les comités d'action lycéens sont apparus lors du mouvement de grève et d'occupation des lycées en mai 1968. Ils sont le cadre commun de débat et d'action des 
lycéens proches de l'extrême gauche. Ils ne survivent pas à la division entre les différents groupes et la Ligue communiste (trotskyste), organisation prépondérante dans ces comités, organise leur dissolution à la rentrée scolaire de 1969. Les lycéens de la Jeunesse communiste, après un bref passage au sein des CAL en juin 68 vont tenter de s'approprier le sigle en créant l'Union nationale des comités d'action lycéens. Cette organisation ne bénéficiera jamais d'une audience suffisante pour contrebalancer l'influence de l'extrême gauche. Elle a été dissoute par le PC à la fin des années 80 .

12. Vincent Gérard 1974. Seuls les trotskystes de l'AJS/OCI et le Mouvement de la jeunesse communiste (MJCF) se sont tenus à l'écart de la coordination.

13. La coordination nationale infirmière éclate définitivement lors d'une assemblée générale à Montpellier le 4 novembre 1989 avec la décision d'une partie des membres de la coordination d'adopter un statut de type syndical.

14. Les CET sont dénommés depuis 1979 Lycées d'enseignement professionnel (LEP).

15. Il nous semble nécessaire de reprendre à notre compte la distinction entre les conseils et les comités de branches opérée par Anweiler 1972 : 45-46 et 138.

16. En ce qui concerne l'analyse des crises politiques, nous nous inspirons des travaux de Dobry 1986.

17. Le CRC est le Comité Régional de Coordination des secteurs santé CFDT de la région parisienne. C'est une structure inhabituelle chargée de coordonner à la fois les syndicats professionnels et départementaux de la région parisienne. Cette structure est animée par des opposants à la majorité confédérale de la CFDT. Les militants du CRC sont exclus par la Confédération à la suite de la grève des infirmières.

18. Irène Leguay (bureau de la coordination infirmière), Collectif, $\mathrm{n}^{\circ}$ 7, Janvier 1989 : 5.

19. Kergoat Danièle 1990.

20. Depuis 1994 et pour la première fois, une des organisations étudiantes, l'Unef SE, est présidée par une femme Marie-Pierre Vieu.

21. On peut souligner que le bureau de la coordination infirmière est mixte, que des infirmiers, en particulier militants du CRC/CFDT, jouent un grand rôle dans l'organisation du mouvement, et Pascal Dias est un des porte-parole reconnus du mouvement.

22. Comme François N., cheville ouvrière du bureau de la coordination, militant de la Ligue communiste, et titulaire d'un DEA.

23. Kergoat $1990: 45$.

24. Ribeill 1987.

25. Bourdieu $1972: 172$.

26. Chez les cheminots, l'étude menée par Georges Ribeill fait aussi apparaître une forte présence de conducteurs se déclarant proches de l'extrême gauche à l'origine des coordinations : « au plan des affinités politiques (toujours en additionnant adhérents et sympathisants) réparties en $36 \%$ pour la Ligue communiste révolutionnaire (effectivement animatrice de la Coordination nationale des agents de conduite durant la grève, Lutte ouvrière étant plutôt animatrice de la Coordination nationale intercatégorielle), 19 \% pour le Parti socialiste, et autant de libertaires divers (UTCL principalement) ». Ribeill 1987.

27. Bourdieu $1980: 114$ et suivantes.

28. INRP 1976

29. Gaxie $1978: 176$

30. Bourdieu $1979: 466$.

31. Lacroix 1980 : 144 et suivantes. 
32. A l'inverse, Isabelle Thomas, étudiante en DEA de Droit des Affaires à Villetaneuse, a un profil en décalage avec le milieu mobilisé. La mise en valeur de ce décalage est un des éléments qui empêchent son élection au bureau de la coordination.

33. Dans le cadre des accords signés par les syndicats, le 24 octobre 1989, une clause prévoit qu'une infirmière chef peut, au bout de 20 ans de carrière, acquérir le rang de médecin au sein de la hiérarchie hospitalière. La durée moyenne de vie professionnelle d'une infirmière en secteur hospitalier est de huit ans. Une des principales filières de sortie est l'établissement comme infirmière indépendante.

34. Leschi 1986.

35. Bennhamias et Leschi 1987.

36. Pour la première fois depuis la fin des années 1950, la première organisation étudiante aux élections universitaires est une organisation corporatiste qui se veut apolitique, la Fédération des associations générales d'étudiants (FAGE).

\section{RÉSUMÉS}

Les coordinations ont fait leur apparition au lendemain de Mai 1968 dans les lycées puis dans les universités. Elles sont devenues un élément constant du répertoire d'action collective des mouvements de jeunesse. Au milieu des années 1980, des coordinations naissent dans des secteurs salariés (cheminots, infirmières, postiers) sans pour autant s'établir durablement comme forme d'action collective. L'apparition de ces coordinations de salariés est à la fois liée aux circonstances et au caractère d'exemplarité qu'a eu la coordination étudiante contre le projet de loi Devaquet; à la présence dans ces secteurs salariés de militants et militantes ayant connu les mobilisations lycéennes et étudiantes des années 70 et remettant en cause les pratiques syndicales traditionnelles, enfin à l'augmentation des compétences des salariés en terme de qualifications et de diplômes. La convergence de ces éléments dans le secteur public explique que les coordinations s'y soient développées. Dans ce cadre, la coordination infirmière n'est ni la figure de mobilisation ni le produit de spécificités propres au genre féminin.

The coordination committees, or « coordinations » as they are known in French, first appeared after the Mai 68 events in high schools, and later in universities. They became one of the central components of the repetoire of collective action of the youth movement. In the mid 80 's, the coordination committees appeared in working class sectors, notably among railworkers, nurses, and post-office agents. Nonetheless, they failed to become institutionalized and to generate longterm collective action. The upsurge of these workers «coordinations" was linked to conjunctural events, but more particularly to their exemplary mobilization of students against the Devaquet Law in 1986. An important factor has been the presence in worker sectors of former university students or high school pupils who participated in protest movements in the 70's, which sought to change traditional forms of trade union action. Lastly, the growth of the coordinations is linked to workers rising qualification and educational levels. The convergence of these factors in the public sector explains why the coordination committees developed. In this sense, the nurses coordination committees are not to be seen as gender-specific but as part of the larger phenomenon discussed in the paper. 


\section{AUTEUR}

\section{DIDIER LESCHI}

Didier LESCHI, après un DEA de politiques comparées sur Un aspect particulier des mobilisations sociales : les coordinations. Étude comparée du phénomène des coordinations, termine une thèse de sciences politiques sur le Parti social français du colonel de La Rocque. Il a collaboré au catalogue sur Mai 68, Les mouvements étudiants en France et dans le monde, (Geneviève DreyfusArmand et Laurent Gervereau, dir.) Nanterre, BDIC, 1988, Il participe aux travaux du Laboratoire d'analyse des systèmes politiques du département de Sciences juridiques, administratives et politiques de l'université de Paris X Nanterre. 